\title{
Radiation recall dermatitis induced by tamoxifen during adjuvant breast cancer treatment
}

\author{
Jiyoung Rhee, MD', Gwi Eon Kim, MD², Chang Hyun Lee, MD³, Jung-Mi Kwon, MD1, \\ Sang-Hoon Han, MD', Young Suk Kim, MD², Woo-Kun Kim, MD \\ Departments of ${ }^{1}$ Internal Medicine, ${ }^{2}$ Radiation Oncology, and ${ }^{3}$ General Surgery, Jeju National University Hospital, Jeju, Korea
}

Tamoxifen and radiotherapy are used in breast cancer treatment worldwide. Radiation recall dermatitis (RRD), induced by tamoxifen, has been rarely reported. Herein, we report a RRD case induced by tamoxifen. A 47-year-old woman had a right quadrantectomy and an axillary lymph node dissection due to breast cancer. The tumor was staged pT2NO; it was hormone receptor positive, and human epidermal growth factor receptor 2 negative. The patient received adjuvant chemotherapy followed by tamoxifen and radiotherapy. After 22 months of tamoxifen, the patient developed a localized heating sensation, tenderness, edema, and redness at the irradiated area of the right breast. The symptoms improved within 1 week without treatment. Three weeks later, however, the patient developed similar symptoms in the same area of the breast. She continued tamoxifen before and during dermatitis, and symptoms resolved within 1 week.

Keywords: Tamoxifen, Radiation recall dermatitis, Treatment

\section{Introduction}

Radiation recall dermatitis (RRD) is an acute skin inflammatory reaction at previously irradiated sites that is induced by the administration of certain agents after radiotherapy [1]. Chemotherapeutic, non-chemotherapeutic agents and a few antibacterials have been reported to induce RRD [1]. Despite several hypotheses about the cause of RRD, its pathophysiology remains poorly understood [2]. Although one study reported that the overall RRD frequency was 8.8\% (8/91) in patients that underwent palliative radiotherapy followed by chemotherapy, other studies have suggested a lower incidence $(<6 \%)[1,3]$.

Tamoxifen and radiotherapy are used in breast cancer treatment worldwide. Tamoxifen-induced RRD, however, has been very rarely reported [4-7]. Therefore, we report a case of
RRD induced by tamoxifen.

\section{Case Report}

A 47-year-old woman was diagnosed with cancer of the right breast in September 2011. The patient underwent quadrantectomy with axillary lymph node dissection. The histopathological analysis revealed a $2.1 \times 2.0-\mathrm{cm}$ invasive ductal carcinoma with no lymph node metastasis. The immunohistochemical assessment of the tumor was estrogen receptor (Allred score, 5) and progesterone receptor (Allred score, 9) positive. The human epidermal growth factor receptor 2 was negative as confirmed by fluorescence in situ hybridization. The patient was administered adjuvant treatment that included four cycles of doxorubicin and cyclophosphamide every 3 weeks, followed by tamoxifen and

Received 9 June 2014, Revised 11 September 2014, Accepted 29 Setember 2014.

Correspondence: Woo-Kun Kim, MD, Division of Hematology-Oncology, Department of Internal Medicine, Jeju National University Hospital, 15 Aran 13-gil, Jeju 690-767, Korea. Tel: +82-64-717-1590, Fax: +82-64-717-1487, E-mail: mwkkim@naver.com

(c) This is an Open Access article distributed under the terms of the Creative Commons Attribution Non-Commercial License (http://creativecommons.org/ licenses/by-nc/3.0/) which permits unrestricted non-commercial use, distribution, and reproduction in any medium, provided the original work is properly cited.

www.e-roj.org 

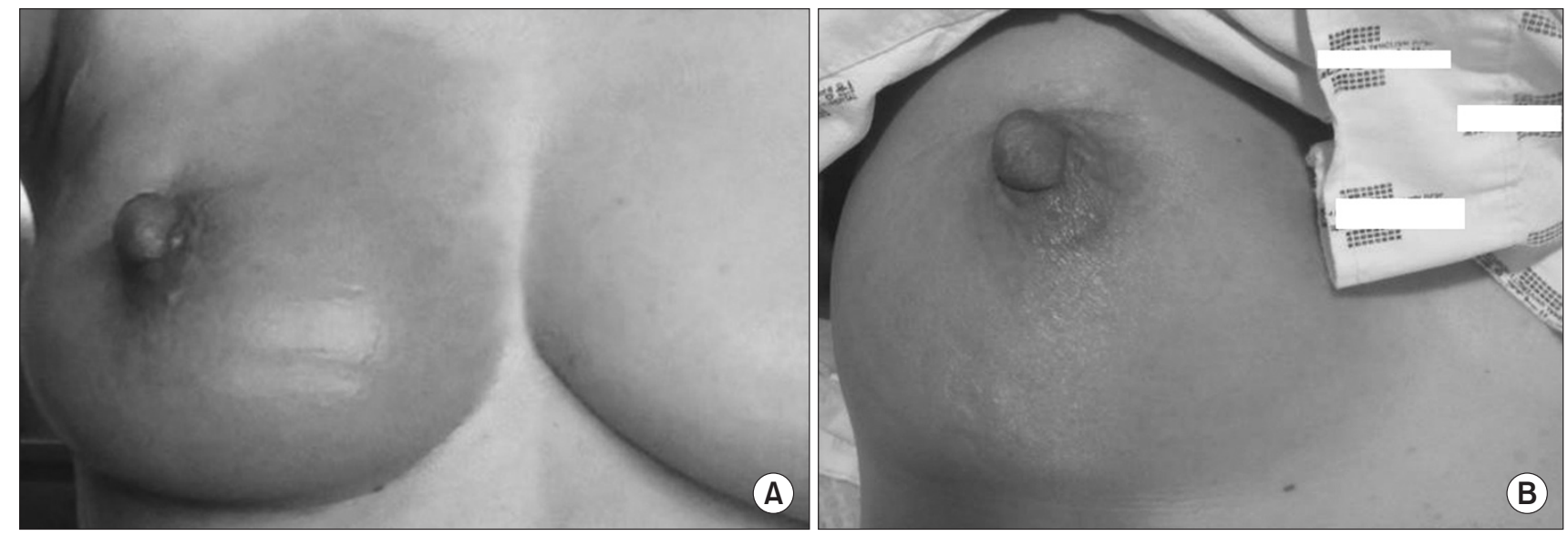

Fig. 1. A well-demarcated skin lesion in a previous irradiated area. The first (A) and second (B) events are shown.

radiotherapy. The patient received 50.4 Gy to her entire right breast, and $10 \mathrm{~Gy}$ locally at the tumor site, over a period of 2 months (between January 17, 2012 and March 19, 2012). Tamoxifen ( $20 \mathrm{mg} /$ day) was also administered to the patient on January 17, 2012.

In December 2013, the patient developed a heating sensation, tenderness, edema, and redness at the irradiated area of the right breast (Fig. 1A). The patient had taken an over-the-counter (OTC) drug for an upper respiratory infection on the previous day. The patient continued taking tamoxifen, and the symptoms subsided within 1 week. In approximately 3 weeks, however, the patient experienced a recurrence of the previous symptoms, the abrupt development of a heating sensation, mild tenderness, edema, and redness, in the same area of the right breast (Fig. 1B). The patient was only taking tamoxifen at this time. The physician empirically prescribed cephalosporins for 14 days under the suspicion of mastitis, despite no obvious signs of infection, no fever or chills, negative blood cultures, and normal C-reactive protein levels. The symptoms improved within 1 week. The patient continued tamoxifen administration.

\section{Discussion}

As a selective estrogen receptor modulator, tamoxifen reduces the recurrence and mortality from hormone receptor (HR)-positive early breast cancer; it is used worldwide as a standard endocrine therapy for HR-positive breast cancer [8]. Tamoxifen-induced RRD has been reported in only four cases since it was first reported in 1992 [4-7]. In prior reports, the patients were diagnosed with tamoxifen-induced RRD based on the following clinical findings: 1) localized symptoms of an acute inflammatory skin reaction at previously irradiated areas, 2) symptom development during tamoxifen treatment, 3) no other therapy provoking RRD, and 4) negative test results for infection and/or breast cancer recurrence or other malignancy [4-7].

Previous studies reported that RRD occurred 5 days to 3 months after tamoxifen administration [4-7]. In two of four reported cases, the symptoms improved in 2 or more weeks after stopping tamoxifen administration. Tamoxifen was administered again to the two patients. They experienced a mild skin reaction only $[4,6]$. The third patient continued tamoxifen during RRD. In this case, the inflammation decreased after 1 months and the affected area completely healed within 3 months [5]. In the case of the last patient, the symptoms gradually improved 7 weeks after stopping tamoxifen; subsequently toremifene was administered [7] (Table 1).

The patient in our study also experienced localized acute inflammatory symptoms in the irradiated area of her right breast without evidence of infection during tamoxifen treatment. The possibility of breast cancer recurrence was excluded due to spontaneous resolving of skin lesion without a change in treatment. Although the patient had taken an OTC drug once prior to the first event, she didn't have any medications except for tamoxifen before the second event. In Korea, an OTC drug does not contain antibiotics, and the patient took antibiotics after her symptoms recurred. Consequently, we excluded the possibility that the antibiotics were the cause of the recurrent RRD in this patient. Additionally, tamoxifen was the only medicine that could have induced recurrent RRD. The patient continued the tamoxifen because we did not realize that the cause of dermatitis was radiation recall phenomenon 


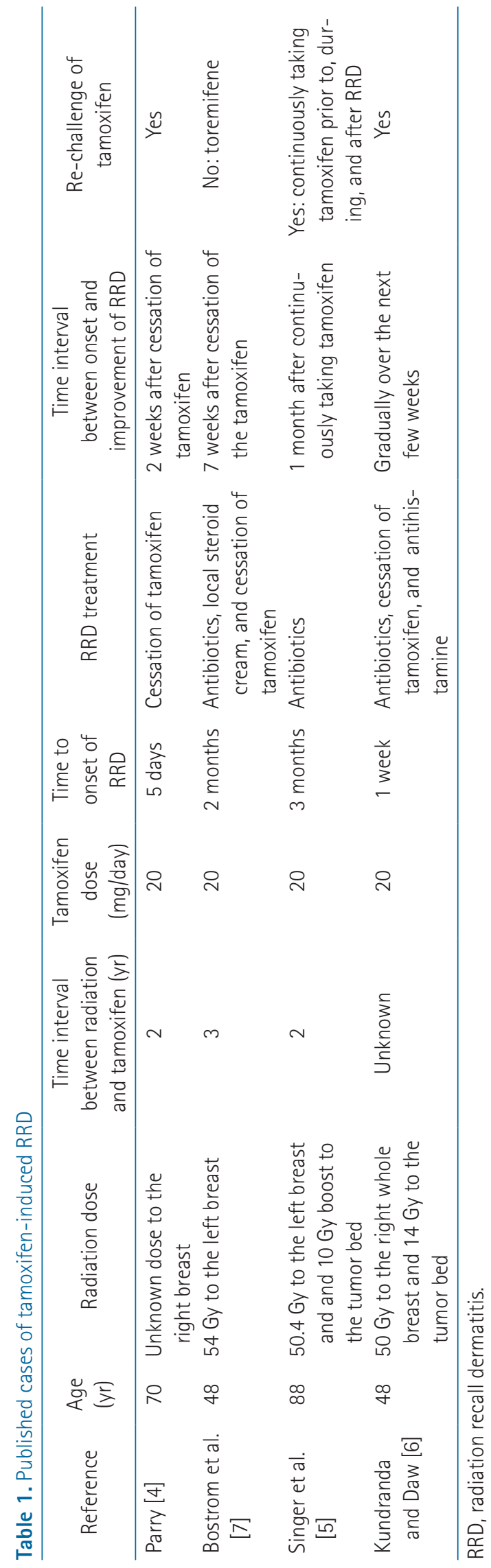

and it could be induced by tamoxifen. However, despite continuing the tamoxifen, the mechanism of spontaneous recovery from RRD in both a previously reported case and in ours is unknown. If we had taken a tissue biopsy from the lesion, it could have helped elucidate the mechanism of this phenomenon.

Until now, guidelines have not existed for diagnosis and treatment of RRD, or for re-challenging patients with precipitating agents $[1,9]$. Previously proposed hypotheses of pathophysiology vary between depletion and/or impaired function of epithelial stem cells, changes in stem cell sensitivity, vascular permeability or proliferative changes, cumulative direct DNA damage and oxidative stress, the Koebner phenomenon, idiosyncratic drug hypersensitivity reactions, and effects of cytokine and mast cells [7,9-16]. However, because these abovementioned hypotheses lack supporting studies, a clinical diagnosis s based on signs, symptoms, laboratory findings (to exclude infection and malignancy), and a history of medication and radiation therapy is the only method to identify patients with RRD.

In our case, the time to RRD onset after tamoxifen administration was 22 months, which is the longest time interval among reported cases of tamoxifen-induced RRD. Although RRD induced by tamoxifen is a rare phenomenon, our case and other reported cases suggest that RRD should be suspected in breast cancer patients with localized dermatitis, such as skin erythema and edema, at the previously irradiated field during tamoxifen treatment. Similar to a previously reported case, our patient also experienced spontaneous RRD improvement while continuing tamoxifen. Therefore, if a patient does not have any reliable option except tamoxifenlike adjuvant treatment in premenopausal HR-positive breast cancer, re-challenging with tamoxifen or continuing the drug may be reasonable even though the patient has experienced tamoxifen-induced RRD. Finally, a better understanding of the pathophysiology of RRD will result in a definite diagnosis without ambiguity using laboratory methods.

In conclusion, a better understanding of the mechanism of RRD is needed to diagnose and manage patients appropriately. Re-challenging or continuing the tamoxifen could be reasonable in premenopausal HR-positive breast cancer patient with mild to moderate degree RRD.

\section{Conflicts of Interest}

No conflict of interest relevant to this article was reported. 


\section{References}

1. Burris HA 3rd, Hurtig J. Radiation recall with anticancer agents. Oncologist 2010;15:1227-37.

2. Hird AE, Wilson J, Symons S, Sinclair E, Davis M, Chow E. Radiation recall dermatitis: case report and review of the literature. Curr Oncol 2008;15:53-62.

3. Kodym E, Kalinska R, Ehringfeld C, Sterbik-Lamina A, Kodym R, Hohenberg $G$. Frequency of radiation recall dermatitis in adult cancer patients. Onkologie 2005;28:18-21.

4. Parry BR. Radiation recall induced by tamoxifen. Lancet 1992;340:49.

5. Singer EA, Warren RD, Pennanen MF, Collins BT, Hayes DF. Tamoxifen-induced radiation recall dermatitis. Breast $J$ 2004;10:170-1.

6. Kundranda MN, Daw HA. Tamoxifen-induced radiation recall dermatitis. Am J Clin Oncol 2006;29:637-8.

7. Bostrom A, Sjolin-Forsberg G, Wilking N, Bergh J. Radiation recall: another call with tamoxifen. Acta Oncol 1999;38:955-9.

8. Early Breast Cancer Trialists' Collaborative Group. Tamoxifen for early breast cancer: an overview of the randomised trials. Lancet 1998;351:1451-67.
9. Camidge R, Price A. Characterizing the phenomenon of radiation recall dermatitis. Radiother Oncol 2001;59:237-45.

10. Hellman S, Botnick LE. Stem cell depletion: an explanation of the late effects of cytotoxins. Int J Radiat Oncol Biol Phys 1977;2:181-4.

11. Seymour CB, Mothersill C, Alper T. High yields of lethal mutations in somatic mammalian cells that survive ionizing radiation. Int J Radiat Biol Relat Stud Phys Chem Med 1986;50:167-79.

12. Smith KJ, Germain M, Skelton H. Histopathologic features seen with radiation recall or enhancement eruptions. J Cutan Med Surg 2002;6:535-40.

13. Wright EG. Radiation-induced genomic instability in haemopoietic cells. Int J Radiat Biol 1998;74:681-7.

14. Camidge R, Price A. Radiation recall dermatitis may represent the Koebner phenomenon. J Clin Oncol 2002;20:4130.

15. Ristic B. Radiation recall dermatitis. Int J Dermatol 2004;43: 627-31.

16. Hymes SR, Strom EA, Fife C. Radiation dermatitis: clinical presentation, pathophysiology, and treatment 2006. J Am Acad Dermatol 2006;54:28-46. 